\title{
A structural examination of agrochemical processing by human carboxylesterase 1
}

\author{
Andrew C. HemmerT ${ }^{\dagger}$ and Matthew R. RedinBo ${ }^{* \dagger, \dagger \dagger}$ \\ ${ }^{\dagger}$ Departments of Biochemistry and Biophysics, and ${ }^{\dagger \dagger}$ Chemistry University of North Carolina at Chapel Hill, Chapel Hill, NC
}

(Received April 13, 2010; Accepted June 1, 2010)

\begin{abstract}
Human carboxylesterase 1 (hCE1) is the primary carboxylesterase expressed in the liver. This critical member of the phase I drug metabolism pathway detoxifies a wide-range of endobiotics, xenobiotics, and agrochemicals. To date, more than a dozen X-ray crystal structures have been elucidated of hCE1 in complex with a broad spectrum of ligands, including organophosphates. These structures provide valuable insights into agrochemical binding and metabolism by hCE1. For example, variable binding pockets that frame the enzyme's catalytic triad and a long, flexible loop capping this region appear to regulate substrate affinity. Stereoisomers of organophosphates illustrate the substrate selectivity of these two pockets. In contrast, pyrethroid isomers likely impact the positioning of the oxyanion hole required to stabilize the negatively charged transition-state oxygen. Finally, it appears that rates of spontaneous hCE1 reactivation in the presence of organophosphates are significantly affected by alkoxy placement within the active site. (C) Pesticide Science Society of Japan
\end{abstract}

Keywords: human carboxylesterase 1, organophosphate binding, pyrethroid metabolism, organophosphate hydrolysis.

\section{Introduction}

The liver drug metabolizing enzyme human carboxylesterase 1 (hCE1, E.C. 3.1.1.1, CES1) is involved in the hydrolysis or transesterification of endo- and xenobiotics, including pyrethroid, carbamate, and organophosphate (OP) agrochemicals (Fig. 1) ${ }^{1-3)}$ Indeed, rats, mice, and some flies are resistant to these toxins due to high levels of carboxylesterase (CE) expression. ${ }^{4,5)}$ This promiscuous enzyme utilizes a Glu-His-Ser catalytic triad in a two-step mechanism to hydrolyze ester, thioester, and amide containing substrates (Fig. 2). ${ }^{2)}$ First, E354 depolarizes $\mathrm{N} \delta 1$ on H468, generating a lone-pair of electrons on the imidazole $\mathrm{N} \varepsilon 2$, which deprotonates S221 for nucleophilic attack on the substrate. This addition generates a transition state where the negatively charged ketone oxygen is stabilized by backbone nitrogen atoms on G142 and G143, referred to as the oxyanion hole. Upon collapse of the first transition state, the leaving group dissociates, forming a stable acyl, phosphonyl, or phosphoryl enzyme intermediate. With

\footnotetext{
* To whom correspondence should be addressed.

E-mail: redinbo@unc.edu

Published online July 26, 2010

(C) Pesticide Science Society of Japan
}

some agrochemicals, such as OPs, this covalent intermediate may result in permanent enzyme inhibition. ${ }^{6,7)}$ In other substrates, such as pyrethroids and carbamates, H468 will deprotonate a water molecule for nucleophilic attack on the covalent intermediate, generating a second tetrahedral transition state. ${ }^{1,8)}$ Following resolution of this state, a newly formed carboxylic, phosphonic, or phosphoric acid is released and the enzyme machinery is regenerated.

There are three rate-regulating kinetic constants associated with this mechanism (Fig. 2). First, the substrate must gain access to the active site; the binding constant $\left(K_{\mathrm{m}}\right)$ describes the affinity of a given ligand, such as an agrochemical, for this

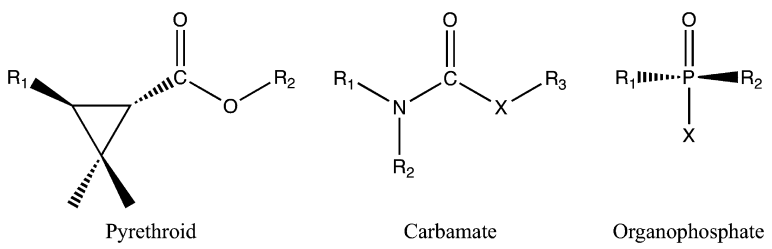

Fig. 1. Chemical structures of agrochemicals processed by hCE1. Organophosphate pesticides contain two $O$-alkoxy groups at $\mathrm{R}_{1}$ and $R_{2}$, while organophosphate nerve agents only contain one. $X$ represents the leaving group in carbamates or OPs, and the alcohol dissociates in pyrethroids. 


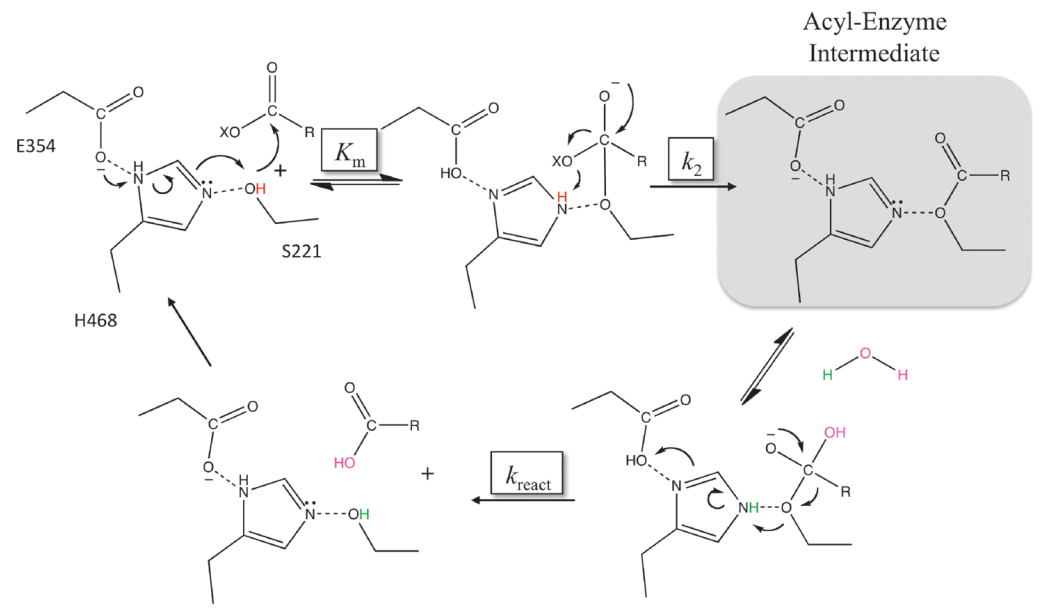

Fig. 2. Catalytic mechanism for carboxyl ester substrate hydrolysis by hCE1. The binding constant $K_{\mathrm{m}}$ defines the affinity of the enzyme for a given substrate and $k_{2}$ describes how quickly the acyl-enzyme intermediate is formed. While pyrethroid pesticides are efficiently hydrolyzed by hCE1, carbamate and OP hydrolysis is often stalled at the acyl-enzyme intermediate formation and may not undergo $k_{\text {react }}$.

pocket. Second, after the initial S221 nucleophilic attack and transition state formation, the rate of acyl, phosphonyl, or phosphoryl enzyme intermediate formation is regulated by $k_{2}$. Third, the rate of hydrolytic attack to finish the catalytic cycle is governed by $k_{\text {react }}{ }^{7)}$

Since 2002, our laboratory has solved thirteen X-ray crystal structures of hCE1 in complex with a variety of ligands, including OP nerve agents. This ensemble of structural information includes various ligand states such as non-covalent, covalently bound, and hydrolyzed substrates within the active site cavity. ${ }^{6,7-11)}$ Here we review structural insights gained from these models to understand hCE1's preferences for agrochemical binding, differences in rates of covalent intermediate formation, and ability of the enzyme to hydrolyze a bound substrate. As this is the primary CE expressed in the liver, it plays a vital role in processing of these toxins. ${ }^{3)}$

\section{Substrate Binding}

The hCE1 catalytic triad is located approximately $18 \AA$ away from the enzyme surface, at the bottom of the active site pocket. These residues are positioned at the interface of catalytic, regulatory, and $\alpha / \beta$ domains and framed by a large, flexible hydrophobic pocket and smaller, rigid, polar pocket. ${ }^{10)}$ Additionally, hCE1 contains a flexible 13-residue acyl-binding loop, which covers the active site, analogous to a lid (Fig. 3).

The structural importance of these two substrate-accommodating pockets is illustrated by differences in binding affinities towards stereoisomers of OPs. It is estimated that approximately $30 \%$ of all OP pesticides on the market exist in racemic mixtures, generally $\mathrm{P}_{R}$ and $\mathrm{P}_{S}$ isomers around the central phosphate. ${ }^{12)}$ In 2007 and 2010, we reported crystal structures of hCE1 in complex with the OP nerve agents soman and cyclosarin, respectively. ${ }^{6,7)}$ hCE1 preferentially bound the $\mathrm{P}_{R}$ isomers of these chemicals, with the large $O$ - pinacolyl and $O$-cyclohexyl groups, respectively, positioned in the open, flexible, hydrophobic binding pocket (Fig. 4). The opposite stereochemistry, which would have orientated the $O$-alkoxy groups into the smaller, rigid pocket, was sterically prohibited.

These structural data were confirmed biochemically by determining bimolecular rates of inhibition $\left(k_{\mathrm{i}}\right)$ of hCE1 for OP nerve agent analogs. ${ }^{7)}$ Utilizing OPs containing a thiomethyl leaving group over the conventional nerve agent fluoride atom, we showed that hCE1 exhibits a 1,700- and 2,900-fold preference for the $\mathrm{P}_{R}$ isomers of soman and cyclosarin mimics. These analogs have been previously shown to form adducts identical to authentic agents. ${ }^{13,14)}$ This second-order inhibition constant $\left(k_{\mathrm{i}}=k_{2} / K_{\mathrm{m}}\right)$ can be broken down into individual components. The rates of phosphonylation $\left(k_{2}\right)$ between $\mathrm{P}_{R}$ and $\mathrm{P}_{S}$ isomers were similar, while the binding constants $\left(K_{\mathrm{m}}\right)$ differed by at least three-orders of magnitude (Fig. 4). The contrast in binding affinities can be attributed to the ability of the larger, flexible binding pocket to accommodate $\mathrm{P}_{R}$ substrates, while large $O$-alkoxy $\mathrm{P}_{S}$ substrates were sterically limited. Indeed, only the smaller $O$-isopropyl in $\mathrm{P}_{S}$ sarin analog readily binds in the smaller, rigid pocket.

This conclusion can be extended to other chiral OP agrochemicals, such as the pesticides diethyl malaoxon, fenofos, or fenamiphos. ${ }^{15-17)}$ In general when considering the binding affinity of a chiral agrochemical in hCE1, a high affinity ligand will be expected to place the large, uncharged structural moiety positioned in the flexible hydrophobic pocket, while smaller groups will be located in the more rigid pocket.

An additional determining factor in agrochemical substrate affinity is the flexible 13-residue acyl-loop capping the active site pocket. The existence and position of the loop is highly variable among esterases. In cholinesterases, such as acetylcholinesterase (AChE) and butyrylcholinesterase, the acylloop dips into the active site pocket and occupies the open hy- 


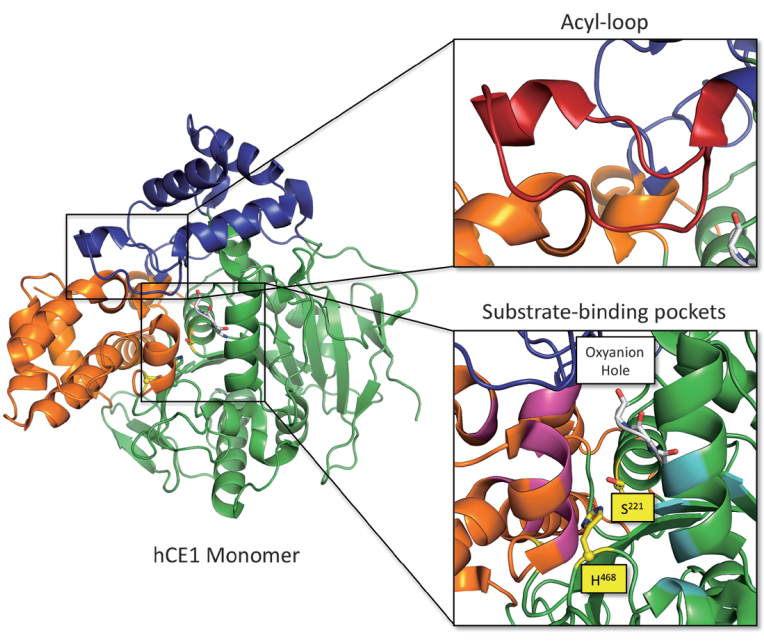

Fig. 3. Architecture of hCE1. hCE1 is composed of three structural domains, an $\sigma / \beta$ (blue), regulatory (orange), and catalytic (green). The E354-H468-S221 catalytic triad (yellow) is located at the interface of these domains. Additionally, the oxyanion hole (white) is part of the catalytic domain. Structural elements that control substrate binding include the long acyl-loop (red inset), which covers the top of the active site, and two pockets that frame the catalytic triad. The large, flexible, hydrophobic pocket (magenta inset) is part of the regulatory domain, while the smaller, rigid, polar pocket (cyan inset) is part of the catalytic domain.
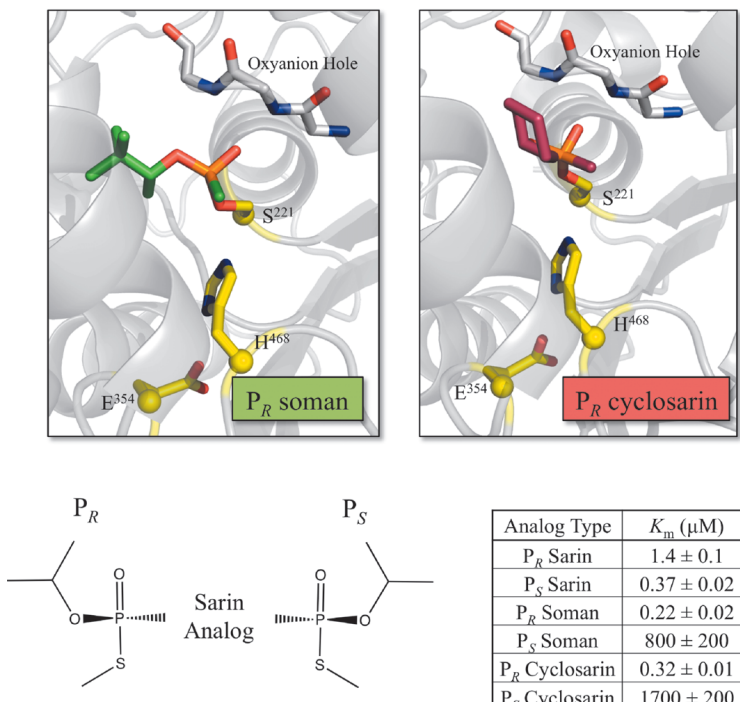

\begin{tabular}{|c|c|}
\hline Analog Type & $K_{\mathrm{m}}(\mu \mathrm{M})$ \\
\hline $\mathrm{P}_{R}$ Sarin & $1.4 \pm 0.1$ \\
\hline $\mathrm{P}_{S}$ Sarin & $0.37 \pm 0.02$ \\
\hline $\mathrm{P}_{R}$ Soman & $0.22 \pm 0.02$ \\
\hline $\mathrm{P}_{S}$ Soman & $800 \pm 200$ \\
\hline $\mathrm{P}_{R}$ Cyclosarin & $0.32 \pm 0.01$ \\
\hline $\mathrm{P}_{S}$ Cyclosarin & $1700 \pm 200$ \\
\hline
\end{tabular}

Fig. 4. X-ray crystal structures of hCE1 and OPs. In both crystal structures, hCE1 preferentially bound the $\mathrm{P}_{R}$ isomers, with the large $O$-alkoxy groups residing in the flexible, hydrophobic pocket. These data were confirmed using thiomethylated OP nerve agent analogs to measure binding constants $\left(K_{\mathrm{m}}\right)$ for these compounds. $\mathrm{P}_{R}$ isomers of soman and cyclosarin bind with $\mathrm{nM}$ affinity, while $\mathrm{P}_{S}$ isomers are poor inhibitors of hCE1. $\mathrm{P}_{S}$ sarin however binds hCE1 very tightly. ${ }^{6,7)}$

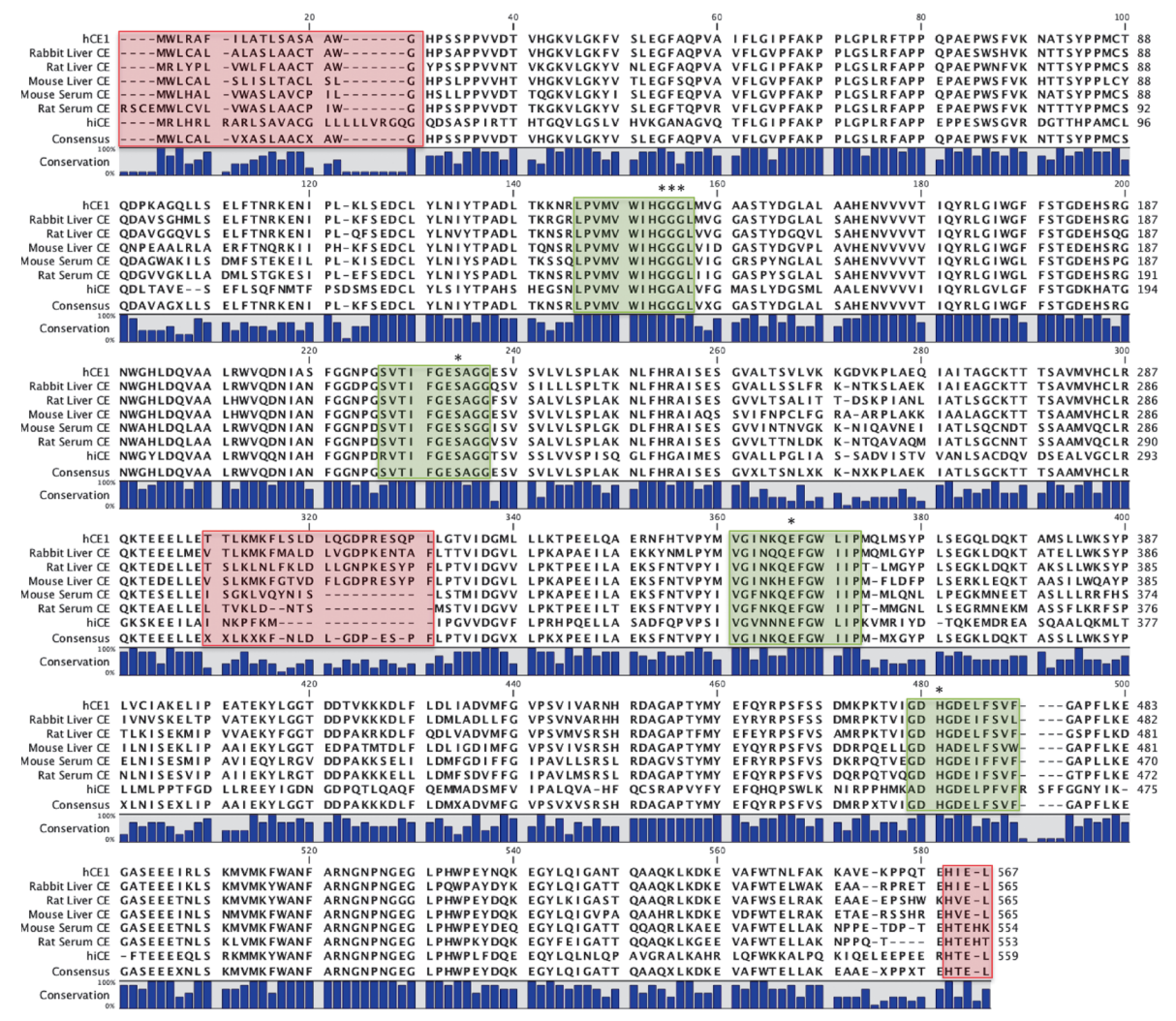

Fig. 5. Multiple sequence alignment of hCE1 with other mammalian CEs. These sequences contain three divergent regions (red): the $N$ - and $C$-terminal localization and retention sequences as well as the acyl-loop at residue 320. Highly convergent regions (green) contain the conserved catalytic residues. 
drophobic region observed in hCE1, thereby greatly affecting substrate stereopreference and binding affinity. ${ }^{6}$ Fig. 5 shows a multiple sequence alignment of hCE1 with other mammalian CEs, including the human intestinal carboxylesterase (hiCE). There are three regions of highly divergent sequences; the $N$-terminal localization sequence, the acyl-loop, and the $C$-terminal retention sequence. The $N$ - and $C$-terminal sequences are enzyme specific and expected to differ between species or cellular localization, but the acyl-loop is unique. ${ }^{18)}$ It is interesting to note that liver CEs contain this loop, while serum proteins and hiCE do not.

\section{Rate of Acyl/Phosphonyl/Phosphorylation}

Following substrate binding, a tetrahedral/pentahedral transition state is formed. The collapse of this unstable state is kinetically described as the rate of acyl-, phosphonyl-, or phosphorylation. ${ }^{7)}$ For effective transition state formation, proper alignment of the growing negative charge on the ketone oxygen with the oxyanion hole is critical. Biochemical and structural investigations of hCE1 with pyrethroid pesticides give insight into the importance of oxyanion hole formation for enzyme acylation and eventual substrate metabolism. ${ }^{19)}$

In 2005, Huang et al. examined structural differences of pyrethroid and fluorescent pyrethroid-like pesticides within the hCE1 active site to explain the different rates of metabolism across stereoisomers. ${ }^{8)}$ Pyrethroids are synthetic analogs of the naturally occurring pyrethrine insecticides, derived from pyrethrum perennials. Compounds such as cypermethrin or fenvalerate are commonly used in large-scale agricultural

applications and consumer products. These agrochemicals contain multiple chiral centers and are primarily metabolized in the liver by esterases, such as hCE1. ${ }^{20)}$ Huang et al. observed that chiral centers in the acid moiety of cypermethrin analogs had a greater impact on hydrolysis than chirality at the alcohol position (Fig. 6). For example, specific activity against trans cypermethrin substrates was greatest, regardless of $R$ or $S$ chirality at the 1 position. hCE1 metabolized these compounds up to 88 -fold more rapidly compared to equivalent cis substrates. Stereoisomers at the alcohol position had minimal effect.

The basis for these varying activities can be explained structurally. The authors manually modeled cis and trans analogs of cypermethrin into the hCE1 active site. Minimal differences in substrate binding were observed between the $1 S$ trans, $\alpha R$ and $1 R$ trans, $\alpha S$ cypermethrin analogs, consistent with the near equivalent enzymatic activities against these compounds. There was a clear difference in these models, however, for substrate binding between $1 S$ cis, $\alpha R$ and $1 S$ trans, $\alpha R$ cypermethrin analogs (Fig. 6). Changing the trans and cis chirality altered positioning of the dichlorovinyl group, thereby causing disruption of the main chain oxyanion hole in cis isomers. Similar clashes were observed with $1 R$ cis-isomers, but not in $1 R$ trans compounds.

The oxyanion hole in hCE1 is formed from backbone nitrogens atoms on G142 and G143 on an extended loop between $\beta$-sheets 6 and $7 .^{10)}$ Based on low crystallographic thermal displacement parameters ( $B$-factors) observed in the available hCE1 crystal structures, this loop is considered rigid, allowing
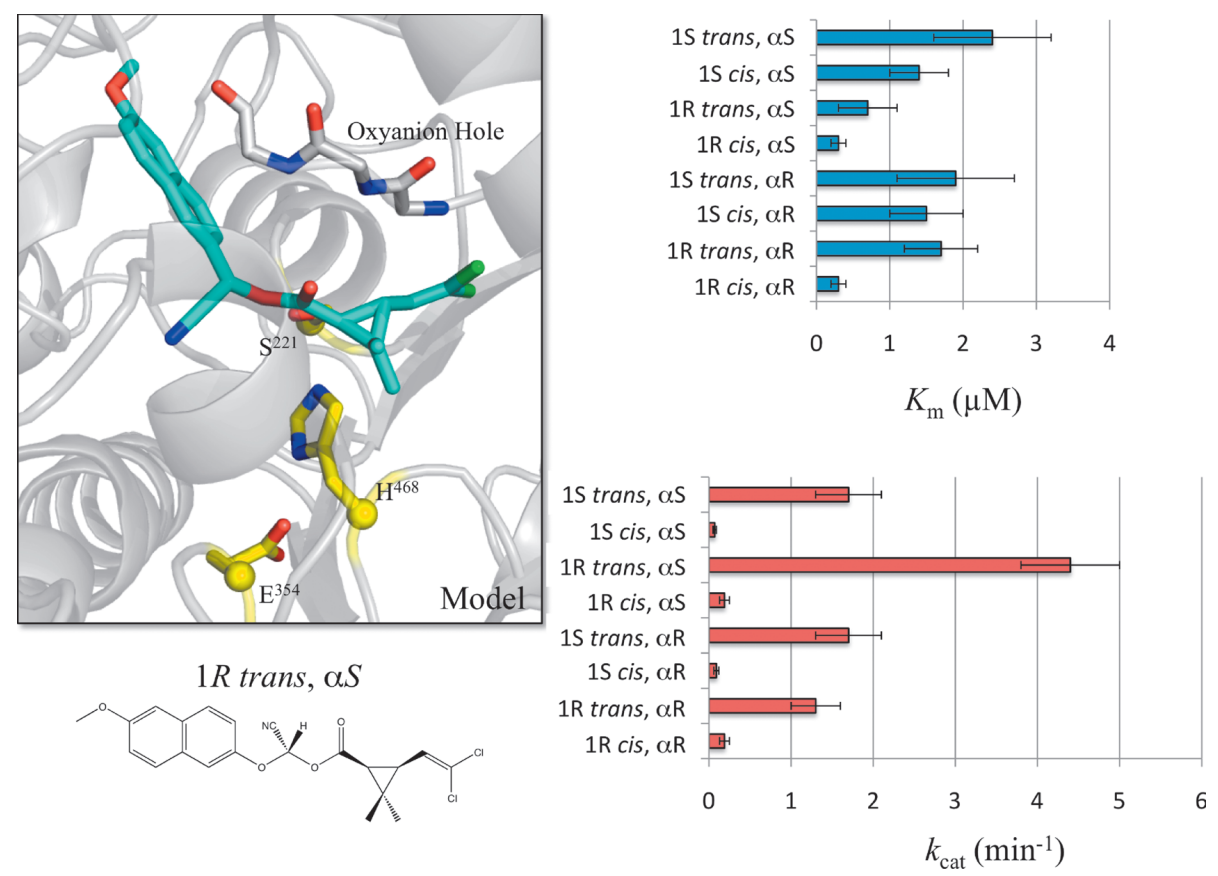

Fig. 6. Cypermethrin metabolism by hCE1. $1 \mathrm{R}$ trans, $\alpha \mathrm{S}$ (cyan structure) is efficiently metabolized, while $1 \mathrm{R}$ cis, $\alpha \mathrm{S}$ is not. Even though cis isomers bind hCE1 more tightly than respective trans isomers, the dichlorovinyl group protrudes into the oxyanion hole, thereby preventing stabilization of the transition state. ${ }^{8}$ 
for minimal movement. ${ }^{11)}$ Failure of hCE1 to effectively metabolize respective cis cypermethrin isomers may be due to disruption in oxyanion hole formation. If the developing negative charge of the first transition state cannot be stabilized, no covalent intermediate will be formed. Indeed, $1 S$ cis cypermethrin analogs actually exhibit tighter binding constants than $1 R$ trans isomers, by at least one-order of magnitude (Fig. 6). The large differences in enzymatic efficiency $\left(k_{\text {cat }} / K_{\mathrm{m}}\right)$ of hCE1 against these stereoisomers stems from $k_{\text {cat }}$, or the ratelimiting step. ${ }^{8)}$ For cis isomers, $k_{\text {cat }}$ may be the rate of acylation.

\section{Rates of Hydrolysis}

Finally, for a number of agrochemicals, such as carbamates and OPs, the decarbamylation or dephosphonylation steps are rate limiting. ${ }^{7,21,22)}$ For example, in $p$-nitrobenzyl esterase (pnb CE), a CE homolog from Bacillus subtilis, the rate of decarbamylation $\left(k_{\text {react }}=3.1 \times 10^{-4} \mathrm{~s}^{-1}\right)$ is 58 -fold slower than $k_{2}$ $\left(0.018 \mathrm{~s}^{-1}\right){ }^{21)}$ Further, as is the case with most OPs, the enzyme may remain permanently phosphonylated, with no hydrolysis occurring. ${ }^{6,7)}$ For example, hCE1 can spontaneously reactivate following racemic sarin inhibition at a rate of $4.8 \times 10^{-6} \mathrm{~s}^{-1}$, but remains permanently inhibited following soman or cyclosarin inhibition. ${ }^{7)}$ A crucial determining factor for hydrolytic attack on a covalent intermediate is the reactive nature of the carbonyl/phosphonyl center. For example, the rate of hydrolysis is drastically reduced in carbamates compared to equivalent carboxylates. It is thought that the nitrogen substitution changes the dipole moment of the central carbonyl carbon by 0.5 Debye units, reducing the partial positive charge on this atom, and possibly the reactivity of the carbamyl-enzyme intermediate. ${ }^{23)}$
Fleming et al. and Hemmert et al. reported and examined X-ray crystal structures of hCE1 in complex with the OP nerve agents soman, tabun, and cyclosarin. ${ }^{6,7)}$ These structures, in addition to stereogenic biochemical data, provide insight into structural constraints that may limit the rates of hydrolysis in OPs. As previously mentioned, hCE1 exhibits no spontaneous hydrolysis of soman or cyclosarin, and the $O$ alkoxy in each agent is positioned into the larger, flexible binding pocket. In 2010, however, we showed that hCE1 can spontaneously reactivation following racemic sarin inhibition. ${ }^{7)}$ Enzyme reactivation studies with thiomethylated OP analogs further demonstrated that hCE1 preferentially hydrolyzed the $\mathrm{P}_{S}$ sarin isomer in racemic mixtures. In contrast, exposure to $\mathrm{P}_{R}$ sarin resulted in permanent enzyme inhibition. $\mathrm{P}_{S}$ sarin positions the $O$-isopropyl group into the smaller, polar binding pocket, which is composed of the catalytic histidine (H468), a neighboring glutamic acid (E220), and a water connected tyrosine (Y152) (Fig. 7A).

There are few possible theories as to why spontaneous hCE1 reactivation is greater for $\mathrm{P}_{S}$ sarin isomers compared to $\mathrm{P}_{R}$. Opposite to carbamates, placing the sarin $O$-isopropyl group into the small polar binding pocket of hCE1 may enhance the dipole moment at the central phosphate, thereby increasing the reactivity of the phosphonate intermediate. The $O$-isopropyl oxygen is $3.7 \AA$ away from $\mathrm{N} \varepsilon 2$ on $\mathrm{H} 468$, increasing the electronegativity of this atom, thereby pulling more charge away from the phosphate. This dipole moment shift would not occur with the equivalent $\mathrm{P}_{R}$ isomer, as the $O$ alkoxy group in the large hydrophobic pocket would make no secondary polar contacts. If the dipole moment on the central phosphate is increased, water may be able to attack this intermediate, resolving the enzyme for another round of catalysis.
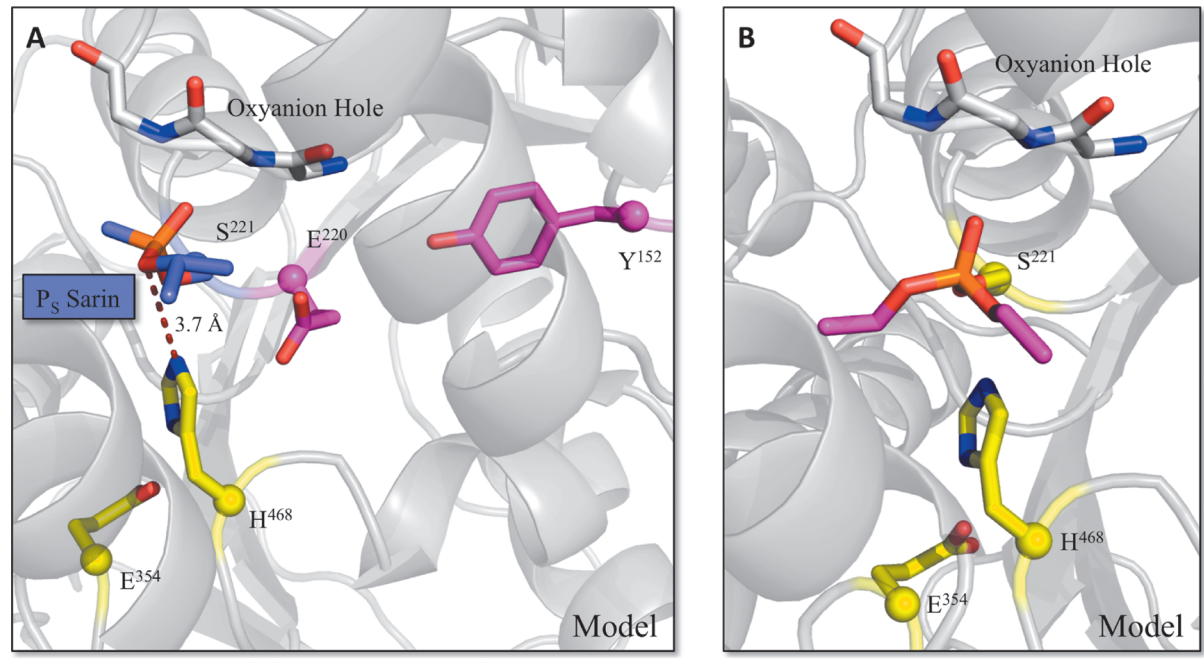

Fig. 7. Organophosphates bound in the hCE1 active site. A) $\mathrm{P}_{S}$ sarin places the $O$-isopropyl group in the smaller, polar, rigid binding pocket, potentially enhancing the dipole moment on the central phosphate. hCE1 can spontaneously reactivate following $\mathrm{P}_{S}$ sarin exposure, but remains inhibited by $\mathrm{P}_{R}$ sarin. B) The diethyl phosphate product following paraoxon inhibition. The equivalent electronegative oxygen atoms bonded around the central phosphate likely neutralize any dipole effects, generating a non-reactive phosphate center, resulting in carboxylesterase inhibition. 
Based on this hypothesis, OP pesticides would be more resistant to hydrolysis than $\mathrm{P}_{S}$ phosphonates. The phosphate center would have a smaller dipole moment because it is linked to equivalently electronegative oxygen atoms. The effect of placing one $O$-alkoxy group into the smaller, polar pocket would most likely not be sufficient enough to overcome the inert phosphate intermediate (Fig. 7B). The literature contains no data regarding hydrolysis of OP pesticides by hCE1; however rat serum CE hydrolyzes paraoxon approximately 9-fold slower than similar branched phosphonates, and muscle CEs in the earthworm Lumbricus terrestris exhibit minimal reactivation following chlorpyrifos inhibition, even after 45 days. ${ }^{5,24)}$

Alternatively, previous work investigating the influence of $\mathrm{pH}$ on spontaneous OP reactivation in AChE identified the importance of two ionizable residues with $\mathrm{p} K_{\mathrm{a}}$ values of 6.9 and 9.8, suggesting possible His and Tyr residues, respectively. ${ }^{25)}$ As mentioned above, in the hCE1, H468 neighbors $\mathrm{P}_{S}$ sarin and Y152 is located approximately $8 \AA$ away. These two residues are connected via a hydrogen-bonding network through Glu220. It has been observed in the structure of the AChE covalent complex with non-aged $\mathrm{P}_{S} \mathrm{VX}$ that the protonated $N \varepsilon 2$ of the catalytic His samples an alternative rotamer to form a hydrogen-bond with E199 (E220 in hCE1) ${ }^{26)}$ If this shift occurs in hCE1 with $\mathrm{P}_{S}$ sarin, the $\mathrm{H} 468 \mathrm{~N} \varepsilon 2$ rotation to E220 would reorient the remaining $\mathrm{N} \delta 1$, formerly hydrogenbonded to E354. It would then be positioned to interact with the $\mathrm{P}_{S}$ sarin $\mathrm{O} 2$ atom and may act as a general base for hydrolytic dephosphonylation. An analogous orientation of the $\mathrm{O} 2$ atom of $\mathrm{P}_{R}$ sarin would not be possible while still maintaining interactions with the oxyanion hole. By this model, hCE1 will only reactivate after inhibition with $\mathrm{P}_{S}$ sarin, consistent with the experimental results presented above.

In general, the rates of hydrolysis in carbamate and OP agrochemicals are limited by the reactive nature of the carbamyl, phosphonyl, or phosphoryl intermediate. While more research needs to be conducted to clarify the mechanism of specific $\mathrm{P}_{S}$ sarin hydrolysis, perhaps enzyme reactivation can be attributed to an enhanced dipole moment on the central phosphate or to an H468 conformational change induced through $O$-alkoxy binding in the small, rigid, binding pocket.

\section{Conclusion}

Based on the extensive structural and biochemical data available for hCE1 with OPs and pyrethroids, we have reviewed substrate binding, rates of covalent intermediate formation, and rates of hydrolysis by this serine hydrolase. The large, flexible, hydrophobic and smaller, rigid, polar binding pockets within the hCE1 active site influence the binding affinity of substrates, including stereoisomers of OP nerve agent analogs. Additionally, a large flexible binding loop found in liver CEs may limit substrate accessibility compared to CEs that do not contain this loop, like hiCE. The rates of acyl, phosphonyl, or phosphorylation are not only affected by the ability of S221 to attack the substrate nucleophilic center, but also by the ability of the oxyanion hole to stabilize the transition state. In hCE1, cis and trans isomers of cypermethrin are metabolized at different rates, possibly due to oxyanion hole disruption in cis enantiomers. Finally, the rates of hydrolysis and enzyme reactivation are related to the reactivity of the acyl, phosphonyl, or phosphoryl centers. It would be expected that for hCE1, pyrethroid pesticides would be most reactive, followed by carbamates and $\mathrm{P}_{S}$ phosphonates. $\mathrm{P}_{R}$ phosphonates and dialkoxy phosphates would be least reactive. With $\mathrm{P}_{S}$ phosphonates, such as $\mathrm{P}_{S}$ sarin, positioning the $O$-isopropyl group into the small, polar binding pocket may enhance the central phosphate dipole moment, thereby enabling hydrolytic attack.

\section{Acknowledgements}

We would like to thank Dr. Tamara Otto and Dr. Doug Cerasoli at the United States Army Medical Research Institute of Chemical Defense, as well as Dr. Monika Wierdl and Dr. Phil Potter at St. Jude Children's Research Hospital for their assistance with the hCE1-nerve agent structures. Additionally, we would like to acknowledge Dr. David Lawrence, Dr. Matt Ross, Dr. Chris Fleming, and Mrs. Monica Frazier for their chemical, biochemical, structural, and computational contributions, respectively, to understanding this carboxylesterase.

\section{References}

1) M. A. Sogorb and E. Vilanova: Toxicol. Lett. 128, 215-228 (2002).

2) M. R. Redinbo and P. M. Potter: Drug Discov. Today 10, 313-325 (2005).

3) M. K. Ross and J. A. Crow: J. Biochem. Mol. Toxicol. 21, 187-196 (2007).

4) R. Heidari, A. L. Devonshore, B. E. Campbell, K. L. Bell, S. J. Dorrian, J. G. Oakeshott and R. J. Russell: Insect Biochem. Mol. Biol. 34, 353-363 (2004).

5) D. M. Maxwell and K. M. Brecht: J. Appl. Toxicol. 21, S103S107 (2001).

6) C. D. Fleming, C. C. Edwards, S. D. Kirby, D. M. Maxwell, P. M. Potter, D. M. Cerasoli and M. R. Redinbo: Biochemistry 46, 5063-5071 (2007).

7) A. C. Hemmert, T. C. Otto, M. Wierdl, C. C. Edwards, C. D. Fleming, M. MacDonald, J. R. Cashman, P. M. Potter, D. M. Cerasoli and M. R. Redinbo: Mol. Pharmacol. 77, 508-516 (2010).

8) H. Huang, C. D. Fleming, K. Nishi, M. R. Redinbo and B. D. Hammock: Chem. Res. Toxicol. 18, 1371-1317 (2005).

9) S. Bencharit, C. L. Morton, J. L. Hyatt, P. Kuhn, M. K. Danks, P. M. Potter and M. R. Redinbo: Chem. Biol. 10, 341-349 (2003).

10) S. Bencharit, C. L. Morton, Y. Xue, P. M. Potter and M. R. Redinbo: Nat. Struct. Biol. 10, 349-356 (2003).

11) C. D. Fleming, S. Bencharit, C. C. Edwards, J. L. Hyatt, L. Tsurkan, F. Bai, C. Fraga, C. L. Morton, E. L. HowardWilliams, P. M. Potter and M. R. Redinbo: J. Mol. Biol. 352, 165-177 (2005).

12) A. W. Garrison: Environ. Sci. Technol. 40, 16-23 (2006).

13) N. H. Barakat, X. Zheng, C. B. Gilley, M. MacDonald, K. 
Okolotowicz, J. R. Cashman, S. Vyas, J. M. Beck, C. M. Hadad and J. Zhang: Chem. Res. Toxicol. 22, 1669-1679 (2009).

14) C. Gilley, M. MacDonald, F. Nachon, L. M. Schopfer, J. Zhang, J. R. Cashman and O. Lockridge: Chem. Res. Toxicol. 22, 1680-1688 (2009).

15) C. Wang, N. Zhang, L. Li, Q. Zhang, M. Zhao and W. Liu: Chirality 22, 612-617 (2009).

16) A. Hassan and W. C. Dauterman: Biochem. Pharamcol. 17, 1431-1439 (1968).

17) P. W. Lee, R. Allahyari and T. R. Fukuto: J. Environ. Sci. Health B 15, 25-37 (1980).

18) P. M. Potter, J. S. Wolverton, C. L. Morton, M. Wierdl and M. K. Danks: Cancer Res. 58, 3627-3632 (1998).

19) K. Nishi, H. Huang, S. G. Kamita, I. H. Kim, C. Morisseau and B. D. Hammock: Arch. Biochem. Biophys. 445, 115-123 (2006).

20) L. C. Gaughan, J. L. Engel and J. E. Casida: Pestic. Biochem.
Physiol. 14, 81-85 (1980).

21) T. M. Streit, A. Borazjani, S. E. Lentz, M. Wierdl, P. M. Potter, S. R. Gwaltney and M. K. Ross: Bio. Chem. 389, 149-162 (2008).

22) S. R. Feaster, K. Lee, N. Baker, D. Y. Hui and D. M. Quinn: Biochemistry 35, 16723-16734 (1996).

23) P. Bruice: "Organic Chemistry," ed. by J. Challice, J. Murdzek, and K. Schiaparelli, Prentice Hall, Upper Saddle River, New Jersey, p. 10, 2001.

24) B. Collange, C. E. Wheelock, M. Rault, C. Mazzia and Y. Capowiez: Environ. Pollut. 158, 2266-2272 (2010).

25) E. Reiner and W. N. Aldridge: Biochem. J. 105, 171-179 (1967).

26) C. B. Millard, G. Kryger, A. Ordentlich, H. M. Greenblatt, M. Harel, M. L. Raves, Y. Segall, D. Barak, A. Shafferman, I. Silman and J. L. Sussman: Biochemistry 38, $7032-7039$ (1999). 